\title{
Tetrasomy 9p Syndrome in a Filipino Infant
}

\author{
Ebner Bon G. Maceda, ${ }^{1,2}$ Erena S. Kasahara, ${ }^{3}$ Edsel Allan G. Salonga, ${ }^{2}$ \\ Myrian R. Dela Cruz and Leniza De Castro-Hamoy ${ }^{1,2}$ \\ ${ }^{1}$ Division of Clinical Genetics, Department of Pediatrics, College of Medicine and Philippine General Hospital, University of the Philippines Manila \\ ${ }^{2}$ Institute of Human Genetics, National Institutes of Health, University of the Philippines Manila \\ ${ }^{3}$ Division of Newborn Medicine, Department of Pediatrics, College of Medicine and Philippine General Hospital, University of the Philippines Manila
}

\begin{abstract}
Tetrasomy $9 p$ syndrome is a rare chromosomal abnormality syndrome whose most common features include hypertelorism, malformed ears, bulbous nose and microretrognathia. These features present as a result of an additional two copies of the short arm of chromosome 9. Here we present a neonate with characteristic facial features of hypertelorism, downslanted palpebral fissure, bulbous nose, small cupped ears, cleft lip and palate, and downturned corners of the mouth. Clinical features were consistent with the cytogenetic analysis of tetrasomy 9p. In general, clinicians are not as familiar with the features of tetrasomy $9 p$ syndrome as that of more common chromosomal abnormalities like trisomies 13,18 , and 21 . Hence, this case re-emphasizes the importance of doing the standard karyotyping for patients presenting with multiple congenital anomalies. Also, this is the first reported case of Tetrasomy 9p syndrome in Filipinos.
\end{abstract}

Key Words: Tetrasomy 9p, isochromosome, hypertelorism, bulbous nose

\section{INTRODUCTION}

Tetrasomy $9 \mathrm{p}$ is a broad term used to describe the presence of a supernumerary chromosome of the short arm of chromosome 9. In more specific terms, the presence of 2 extra copies of $9 p$ arms can be either isodicentric or pseudodicentric. Isodicentric chromosome is an isochromosome which includes two centromeres linked by a single centromere region. Pseudodicentric, on the other hand, refers to the presence of 2 centromeres, but only one is active. In this variety of tetrasomy $9 p$, the 2 extra copies of $9 p$ are linked by a proximal segment of $9 \mathrm{q}$, which may incorporate euchromatic material. ${ }^{1}$

Tetrasomy $9 p$ leads to a broad range of phenotypic features from multiple congenital anomalies with severe growth retardation and intellectual disability to subnormal cognitive and physical development. The most common features include low birth weight, severe retardation, large anterior fontanelle, hypertelorism, beaked or bulbous nose, cleft lip and palate, and low-set and malformed ears. Just like the other chromosomal abnormalities, like trisomies 13,18 , and 21 , tetrasomy $9 \mathrm{p}$ syndrome, is also diagnosed by standard and molecular cytogenetic investigations like standard karyotyping and chromosomal microarray. ${ }^{1,2}$

We present the clinical features of a Filipino infant with

Corresponding author: Ebner Bon G. Maceda, MD

Division of Clinical Genetics

Department of Pediatrics

Philippine General Hospital

University of the Philippines Manila

Taft Avenue, Manila 1000, Philippines

Email: egmaceda@up.edu.ph tetrasomy $9 \mathrm{p}$ and compare our findings with those of the previously described individuals with the same cytogenetic picture. There are no reported cases and prevalence studies yet of this chromosomal abnormality in the Philippines. Worldwide, less than 60 cases have been reported in over 
40 years. Prenatal diagnosis of tetrasomy $9 p$ is estimated to be $0.002 \% .^{1,3}$ In this report, the value of standard karyotyping or chromosomal analysis in patients presenting with multiple congenital anomalies is re-emphasized.

\section{CLINICAL CASE}

A full term baby girl was born 38 weeks by pediatric aging, with a birth weight of 2495 grams, via low segment caesarean section to a 28 -year-old gravida 4, para 2 mother. The patient was the third of a sibship of three born to a healthy non-consanguineous couple of Filipino descent. The family history was non-contributory (Figure 1).

Congenital anomaly scan done at 22 weeks age of gestation showed vermian agenesis, hydrocephalus, hypertelorism, bilateral cleft lip and palate, cardiomegaly, pleural and pericardial effusion, probably atrial septal defect, and Dandy-Walker Malformation.

On physical examination, the patient was proportionately small for age. The weight is 2495 grams $\left(<10^{\text {th }}\right.$ percentile), length is $45 \mathrm{~cm}\left(<10^{\text {th }}\right.$ percentile $)$ and head circumference is $30.5 \mathrm{~cm}\left(<10^{\text {th }}\right.$ percentile). She had a large anterior fontanelle, absent eyebrows, hypertelorism, downslanted palpebral fissure, bulbous nose, small cupped ears, cleft lip and palate, downturned corners of the mouth, short neck, skin tag on the xiphoid area, brachydactyly with hypoplastic nails with decreased creases on both upper extremities, and a proximally inserted fourth digit on the left lower extremity (Figures 2 and 3).

Postnatal echocardiography done revealed a perimembranous ventricular septal defect, patent ductus areteriosus, patent foramen ovale, severe tricuspid regurgitation, right ventricular hypertrophy, persistent left superior vena cava, severe pulmonary artery hypertension with good biventricular contractility.

Standard karyotyping, which analyzes lymphocytes in the metaphase, revealed an abnormal female chromosome complement in all cells examined with an extra pseudo isodicentric chromosome 9, containing the short arm to the long arm at band q21.1 of chromosome 9, resulting in tetrasomy of chromosome 9pter to 9q21.1 (Figure 4).

The patient needed ventilator support and was managed at the neonatal intensive care unit. On the $5^{\text {th }}$ day of life, she had clinical and radiographic signs of pulmonary congestion, hence was started on furosemide. A repeat chest radiograph on the $16^{\text {th }}$ day of life revealed bilateral pleural effusion and cardiomegaly. She was eventually started on dobutamine, enalapril, spironolactone and hydrochlorothiazide. There was difficulty in weaning from the mechanical ventilator until she developed severe pulmonary hypertension and nosocomial infection leading to her demise on the $32^{\text {nd }}$ day of life.

Pre-test counselling and post-test genetic counselling were done. Appropriate informed consent was obtained from the patient's parents to publish this case and use the patient's photographs.

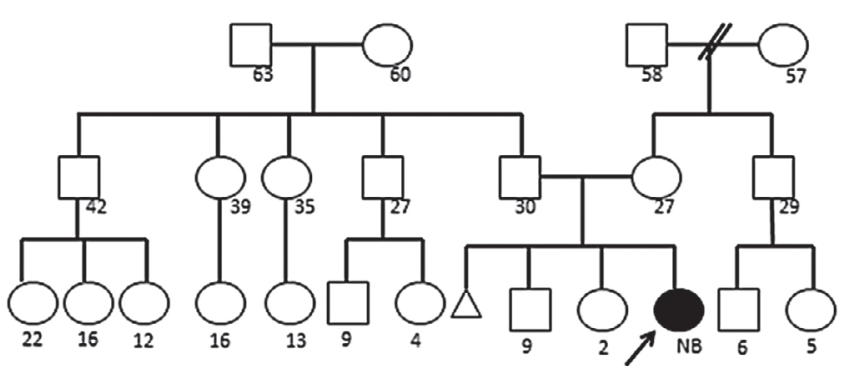

Figure 1. The three-generation family medical history of the patient.
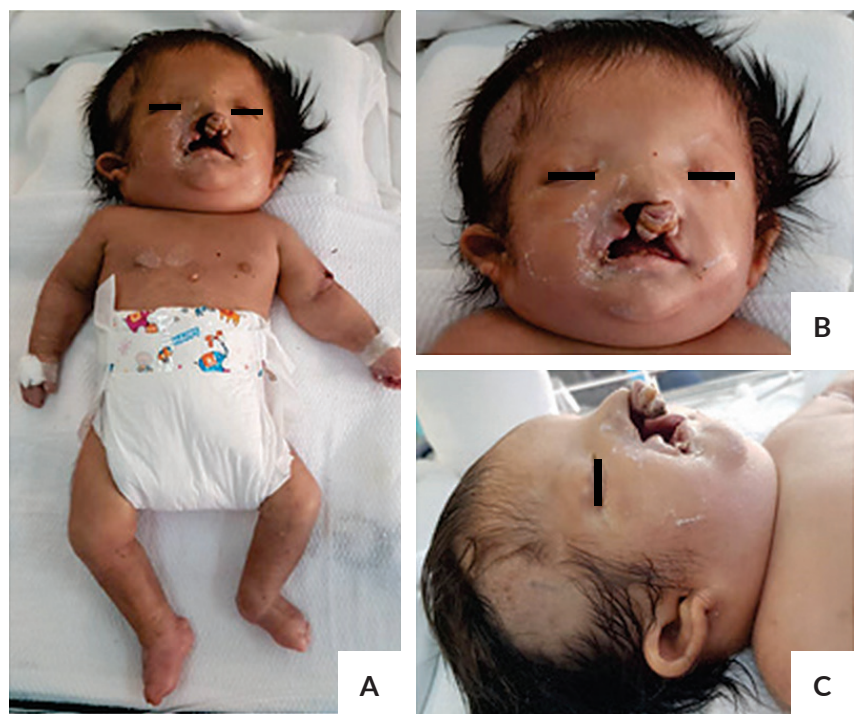

Figure 2. Absent/sparse eyebrows, hypertelorism, downslanted palpebral fissure, bulbous nose, small cupped ears, cleft lip and palate, downturned corners of the mouth, skin tag on the xiphoid area.
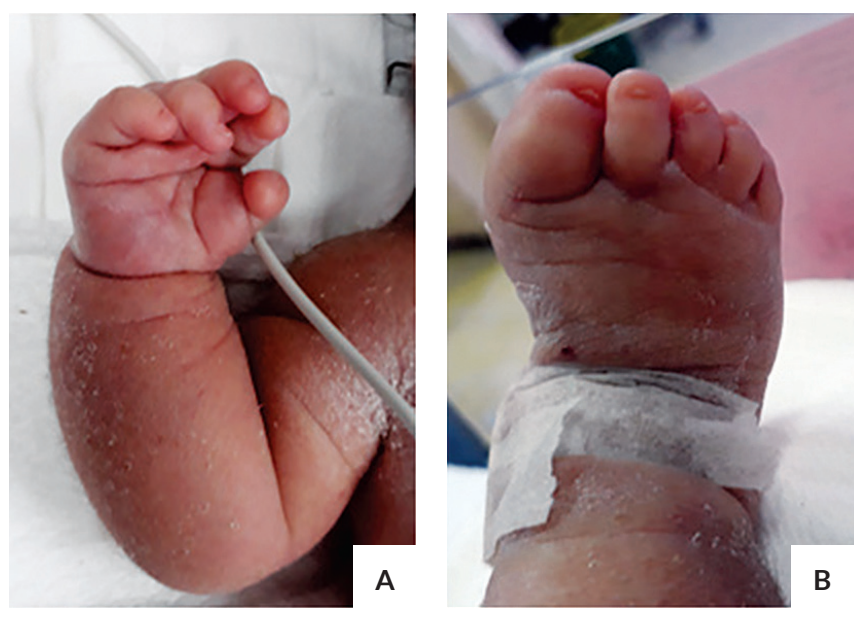

Figure 3. Brachydactyly with hypoplastic nails with decreased creases on both upper extremity $(A)$. 


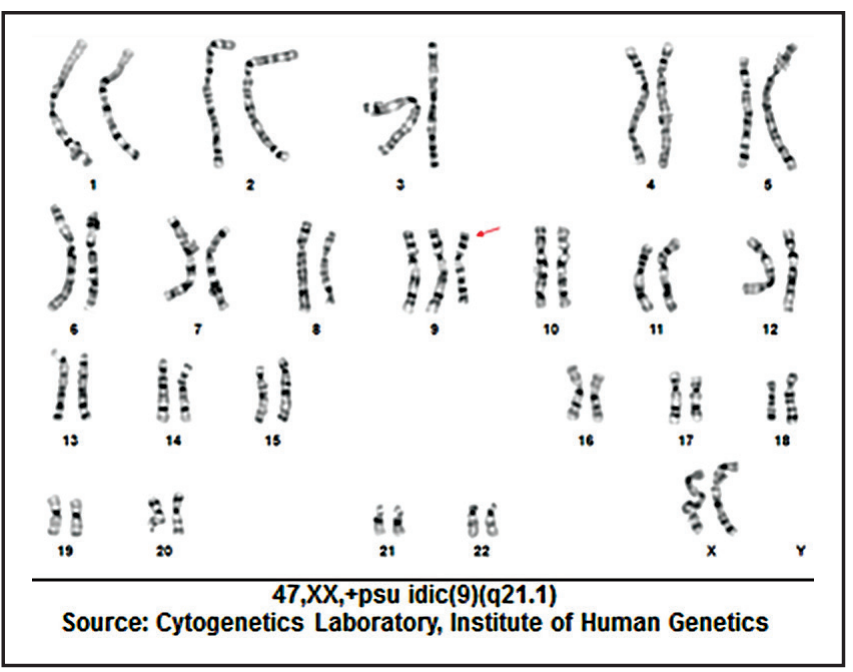

Figure 4. The chromosomal analysis with a supernumerary pseudodicentric chromosome 9.

\section{DISCUSSION}

Phenotypic expression of tetrasomy $9 \mathrm{p}$ cases is broad, however, there are sufficient common features that could lead us to this consideration in many cases (Table 1). Characteristic facial features, as reported in earlier publications, include hypertelorism ( $72 \%$ of 32), bulbous nose (69\% of 32), cleft lip and/or palate (78\% of 32), ear anomalies (88\% of 32), and micrognathia (59\% of 32). Other features include central nervous system anomaly (89\% of 27), limb defects (88\% of 32), congenital heart disease (62\% of 29), small gestational age (57\% of 30), postnatal growth failure $(71 \%$ of 14), developmental delay ( $94 \%$ of 17 ), renal anomalies

Table 1. Comparison of Clinical Features of our patient with the previously reported cases of Tetrasomy $9 p$

\begin{tabular}{lc}
\multicolumn{1}{c}{$\begin{array}{c}\text { Clinical features in previously reported } \\
\text { cases of Tetrasomy 9p Syndrome }\end{array}$} & $\begin{array}{c}\text { Features present } \\
\text { in our Patient }\end{array}$ \\
SGA/IUGR & $(+)$ \\
Early Death & $(+)$ \\
CNS Anomaly & $(+)$ \\
Brachycephaly & - \\
Wide sutures/large fontanelle & $(+)$ \\
Hypertelorism & $(+)$ \\
Microphthalmia & - \\
Epicanthal folds & - \\
Downslanted palpebral fissures & $(+)$ \\
Prominent nasal bridge/bulbous or beaked nose & $(+)$ \\
Ear anomaly & $(+)$ \\
Cleft lip/palate/high arched palate & $(+)$ \\
Downturned corners of mouth/short philtrum & $(+)$ \\
Micro/retrognathia & - \\
Cardiovascular anomaly & $(+)$ \\
Limb contractures/joint dislocation & - \\
Other limb defects & $(+)$ \\
Vertebral/spinal abnormality & - \\
Renal anomaly & - \\
Gl anomaly & - \\
Abnormal genitalia & - \\
\hline
\end{tabular}

( $57 \%$ of 23 ), wide sutures/large fontanelle (56\% of 32), and short neck/excess nuchal skin (53\% of 32$).{ }^{4}$ Majority of these features are seen in our patient.

Even prenatally, findings of the congenital anomaly scan were consistent with tetrasomy $9 \mathrm{p}$ syndrome. Previously reported cases of tetrasomy $9 \mathrm{p}$ show notable sonographic findings which include the following: cleft lip/palate, DandyWalker malformation, limb malformation, intrauterine growth restriction, ventriculomegaly, and genitourinary anomaly. ${ }^{5}$ Of which, ventriculomegaly and Dandy-Walker malformation (60\%) and cleft lip/palate (55\%) are the most frequently reported anomalies. In some cases, however, facial dysmorphism may be too subtle to suggest the diagnosis. ${ }^{1}$ For our patient, the presence of Dandy-Walker malformation, cleft lip and palate, and a congenital heart disease were noted. However, these features may be seen even in other cases with or without chromosomal abnormality. DandyWalker malformation can be seen frequently in craniocerebello-cardiac syndrome and Walker-Warburg syndrome, and occasionally in a lot of conditions including trisomy 9 syndrome, XXXXX syndrome and XYY syndrome. Cleft lip and palate can be seen frequently in many conditions like fetal hydantoin syndrome, microdeletion 2q31.1 syndrome, deletion $4 \mathrm{p}$ syndrome, and Fryns syndrome. Congenital heart disease can be seen in many chromosomal abnormality syndromes like deletion $9 \mathrm{p}$ syndrome, and duplication $3 \mathrm{q}$ syndrome. It can also be seen in other conditions not associated with a chromosomal abnormality like VACTERL Association. ${ }^{6}$ Hence, standard karyotyping in cases of multiple congenital anomalies may aid clinicians in determining the presence of chromosomal aberration.

Clinical genetic testing, such as standard karyotyping, is common practice for patients with unexplained developmental delay, autism spectrum disorders, and multiple congenital anomalies. The utility of chromosomal microarray (CMA) in more advanced settings is now more common since it has a much higher diagnostic yield than the standard karyotyping. CMA offers similar function but at a much higher resolution for genomic imbalances than the standard karyotyping. However, standard karyotyping still offers value in obvious chromosomal syndromes, a family history of chromosomal rearrangement, or in a history of multiple miscarriages. ${ }^{7}$

Severity of phenotype in tetrasomy $9 \mathrm{p}$ syndrome is also variable. The presence of significant defects of the brain, heart, and kidney dictates the prognosis of these cases. ${ }^{1}$ In the literature, these findings are more frequent in non-mosaics than mosaics, and in pseudodicentric cases than in isodicentric cases. ${ }^{4}$ Furthermore, early infant deaths are associated with non-mosaics. Based on our patient's chromosomal analysis, she is a non-mosaic and a pseudodicentric. Pseudodicentric chromosome has only one functional centromere which is morphologically identified as one primary constriction, as noted in this case (Figure 5). Severity of phenotype is influenced by the size of the involved 

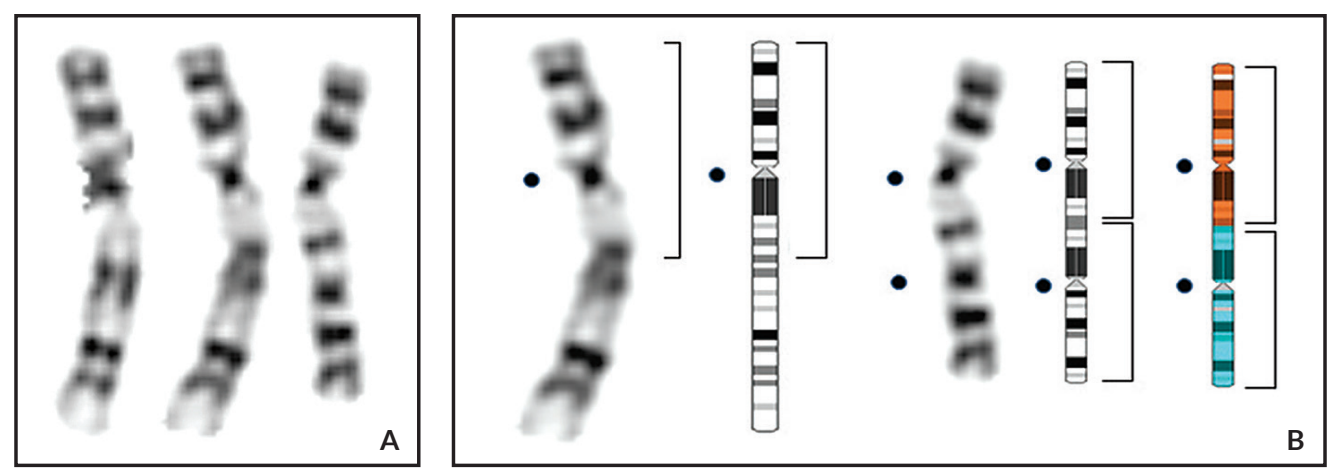

Figure 5. (A) shows a more focused picture of the supernumerary pseudodicentric chromosome 9 and (B) an ideogram showing the position of the centromere represented by a dot. It can be seen that the pseudodicentric chromosome 9 has only 1 primary constriction.

isochromosome, the degree of mosaicism and also the presence of tissue mosaicism. ${ }^{3}$ For instance, the presence of cleft lip and palate was also observed to occur if $i(9 p)$ is found in another tissue aside from the blood tissue. ${ }^{1}$

The contribution of the position of chromosomal breakpoints has also been investigated. The involvement of $9 \mathrm{q}$ region, as observed in previous reports, is more often associated with cardiac malformations, intellectual disability, and death. ${ }^{1,8}$ The chromosomal breakpoint at q21 in this case contributes to the poor prognosis.

Genetic counselling for families with tetrasomy $9 p$ is important. It is usually sporadic, hence the chance of recurrence is not increased. However, there is still a risk for familial supernumerary marker chromosome. It is possible that parents carry the marker but have a normal phenotype and have a severely affected child. Parental karyotyping may be done to show this, as well as to exclude translocation. ${ }^{9}$ For this family, parental karyotyping was offered but was not done.

Management of patients with tetrasomy $9 p$ depends on the associated anomalies, as well as the clinical picture. Early intervention programs may be done for those presenting with mild developmental delay and minor anomalies. This include a wide array of services like physical, occupational and speech therapies aiming to enhance the competence of patients in all developmental domains in order to prevent or minimize delays. ${ }^{10,11}$ For severe cases with early death, such as in our case, supportive care may be given. ${ }^{11}$

\section{CONCLUSION}

We are presented with a case of a neonate with multiple congenital anomalies, initially detected prenatally. Although clinical features and cytogenetic profile confirmed tetrasomy $9 p$ syndrome, the familiarity of clinician, in general, with the features of this syndrome is not as keen as with the common chromosomal abnormalities like trisomy 13, 18 and 21. With this case, the importance of doing standard chromosomal analysis in babies with multiple congenital anomalies is re-emphasized.

\section{Statement of Authorship}

All authors have approved the final version submitted.

\section{Author Disclosure}

All authors declared no conflicts of interest.

\section{Funding Source}

None.

\section{REFERENCES}

1. Khattabi LE, Jaillard S, Andrieux J, Pasquier L, Perrin L, Capri Y, et al. Clinical and molecular delineation of tetrasomy $9 p$ syndrome: report of 12 new cases and literature review. Am J Med Genet A. 2015 Jun; 167(6):1252-61.

2. Jalal SM, Kukolich MK, Garcia M, Benjamin TR, Day DW. Tetrasomy 9p: an emerging syndrome. Clin Genet. 1991 Jan; 39(1): 60-4.

3. Chen CP, Wang LK, Chern SR, Wu PS, Chen YT, Kuo YL, et al. Mosaic tetrasomy 9p at amniocentesis: Prenatal diagnosis, molecular cytogenetic characterization, and literature review. Taiwan J Obstet Gynecol. 2014 Mar; 53(1):79-85

4. Dhandha S, Hogge WA, Surti U, McPherson E. Three cases of tetrasomy 9p. Am J Med Genet. 2002 Dec; 113(4):375-80.

5. Nakamura-Pereira M, do Cima LC, Llerena JC, Guerra FAR, Peixoto-Filho FM. Sonographic findings in a case of tetrasomy $9 p$ associated with increased nuchal translucency and Dandy-Walker malformation. J Clin Ultrasound. 2009 Oct; 37(8):471-4.

6. Jones KL, Jones MC, Del Campo M. Smith's Recognizable Patterns of Human Malformation, 7th Edition. Philadephia: Elsevier; 2013.

7. Miller DT, Adam MP, Aradhya S, Biesecker LG, Brothman AR, Carter NP, et al. Consensus statement: chromosomal microarray is a first-tier clinical diagnostic test for individuals with developmental disabilities or congenital anomalies. Am J Hum Genet. 2010 May; 86(5):749-64.

8. Duzcan F, Ergin H, Aytan M, Tepeli E, Ozdemir O, Basaran S. Non-mosaic tetrasomy $9 \mathrm{p}$ in an infant with multiple congenital anomalies. Gynecol Obstet Reprod Med. 2007; 13(2):114-6.

9. Firth HV, Hurst JA. Oxford Desk Reference Clinical Genetics and Genomics, 2nd Edition. Oxford University Press; 2017. pp. 690-691.

10. Majnemer A. Benefits of early intervention for children with developmental disabilities. Semin Pediatr Neurol. 1998 Mar; 5(1):62-9.

11. Chen H. Atlas of Genetic Diagnosis and Counseling. Humana Press Inc.; 2006. pp. 947-949. 